Pacific Journal of Mathematics

TORSION FREE ABELIAN GROUPS QUASI-PROJECTIVE 


\section{TORSION FREE ABELIAN GROUPS \\ QUASI-PROJECTIVE OVER THEIR ENDOMORPHISM RINGS}

\section{Vinsonhaler and W. J. Wickless}

Certain classes of torsion free abelian groups which are quasi-projective as modules over their endomorphism rings are characterized. The main results concern completely decomposable and strongly indecomposable groups.

1. Preliminaries. Abelian groups which are quasi-projective over their endomorphism rings have been characterized by Fuchs in the torsion case. His methods have been extended by Longtin to the algebraically compact and cotorsion groups [5]. In this paper, we investigate some other classes of groups with this property. Specifically:

Definition. A (left) module $M$ over a ring $R$ is quasi-projective provided the natural map $\operatorname{Hom}_{R}(M, M) \rightarrow \operatorname{Hom}_{R}(M, M / K)$ is epic for every submodule $K$, of $M$.

An abelian group $G$ will be called $E$-quasi-projective (Eqp) provided $G$ is quasi-projective as a module over $E=$ End $(G)$. Henceforth, the word group will denote a torsion free abelian group. Other notation follows Fuchs [4], in particular, $t(G)=$ type $G$ for any group $G$ of rank 1 .

The following simple lemmas will be quite useful.

LEMMA 1.1. Let $G$ be Eqp and $K$ a fully invariant subgroup of $G$. Then $G / K$ is a quasi-projective E-module.

Proof. See Proposition 2.1 in Wu and Jans [9].

LEMMA 1.2. Let $G$ be Eqp and $K$ a fully invariant subgroup. Then $Z E$, the center of $E$, maps onto $\operatorname{Hom}_{E}(G / K, G / K)$.

Proof. Let $\Pi: G \rightarrow G / K$ be the factor map. Since $G$ is Eqp, for every $\theta \in \operatorname{Hom}_{E}(G / K, G / K)$, there exists $\alpha \in \operatorname{Hom}_{E}(G, G)=Z E$ such that $\Pi \alpha=\theta \Pi$.

LEMMA 1.3. Let $G$ be Eqp and $K$ a fully invariant subgroup such that $G / K$ is torsion. Then if $Z E$ is countable, $G / K$ is bounded. 
Proof. If $G / K$ is unbounded and torsion, then $\operatorname{Hom}_{E}(G / K, G / K)$ is uncountable: it must contain either a copy of $Q_{p}^{*}$ (the ring of $p$-adic integers) for some prime $p$, or a direct product of an infinite number of cyclic groups. Hence the result follows from Lemma 1.2.

2. Decomposable groups. In this section, some classes of decomposable Eqp groups are characterized, including completely decomposable and homogeneous separable groups.

We begin with completely decomposable groups, those groups $G$ isomorphic to a direct sum of rank one groups.

LeMma 2.1. If $G=\bigoplus_{\imath \in I} \Sigma A_{\imath}$ is a direct sum of rank one groups, then $G$ is indecomposable as an $E$-module if and only if given any two summands $A_{i}$ and $A_{j}$, there is a finite sequence $A_{\imath}=A_{i_{1}}, A_{i_{2}}, \cdots, A_{i n}=A_{l}$ such that $t\left(A_{l k}\right)$ is comparable to $t\left(A_{l_{k+1}}\right)$ for $k=1,2, \cdots, n-1$.

Proof. If $S$ is a subset of the set $\left\{A_{i}\right\}_{l \in I}$, define $I(S)=\left\{A_{l} \mid t\left(A_{l}\right)\right.$ is comparable to $t\left(A_{m}\right)$ for some $A_{m}$ in $\left.S\right\}$ and $I^{n}(S)=I\left(I^{n-1}(S)\right)$. Then it is easy to see that for a fixed $A_{w}, \oplus \Sigma\left\{A_{\imath} \mid A_{\imath} \in \bigcup_{n=1}^{\infty} I^{n}\left(A_{w}\right)\right\}$ and $\oplus \Sigma\left\{A_{i} \mid A_{l} \notin \cup_{n=1}^{\infty} I^{n}\left(A_{\iota}\right)\right.$ are $E$-submodules whose sum is $G$. The lemma follows immediately.

LEMma 2.2. If $G=\oplus \Sigma A_{t}$ is completely decomposable and indecomposable as an $E=\operatorname{End}(G)$ module, then $Z E \subseteq Q$.

Proof. Maps in $Z E$ must commute with projections and maps $A_{i} \rightarrow A_{r}$. The fact that $G$ is $E$-indecomposable and Lemma 2.1 imply that any map in $Z E$ multiplies each $A_{t}$ by the same rational number.

Theorem 2.1. Let $G=\bigoplus_{i \in I} A_{l}$ be a direct sum of rank one groups such that $G$ is indecomposable as an $E$-module. Then the following are equivalent:

1. $G$ is Eqp.

2. The type set $T=\left\{t_{t} \mid t_{t}=t\left(A_{\imath}\right)\right.$ for some $\left.i\right\}$ satisfies:

(a) If $t_{t}, t_{j} \in T$ and $t_{t}, t_{j} \leqq t_{k}$ for some $t_{k} \in T$, then $t_{t}, t_{l} \geqq t_{l}$ for some $t_{l} \in T$

(b) Countable descending chains in $T$ are bounded below;

(c) If $t_{i} \in T$ is finite at an infinite set of positions $\left\{p_{j}\right\}$, then $\exists t_{k} \in T$ such that $t_{k}$ is 0 at an infinite subset of $\left\{p_{l}\right\}$.

3. If $K$ is a fully invariant subgroup of $G$ such that $G / K$ is torsion, then $G / K$ is bounded.

Proof. (1) $\Rightarrow(2)$. Let $t_{i}, t_{j}, t_{k} \in T$ such that $t_{i}, t_{l} \leqq t_{k}$. Suppose 
there is no $t_{l}$ such that $t_{l} \leqq t_{l}, t_{j}$. Let $K=\bigoplus \Sigma\left\{A_{m} \mid t_{m} \not t_{l}\right.$ and $\left.t_{m} \not t_{j}\right\}$. Then $K$ is a fully invariant subgroup of $G$, and $G / K$ is a direct sum of two $E$-modules, $G / K=B_{1} \oplus B_{2}$ where $B_{1}=\bigoplus \Sigma\left\{A_{m} \mid t_{m} \leqq t_{i}\right\}+K$ and $B_{2}=\bigoplus \Sigma\left\{A_{m} \mid t_{m} \leqq t_{\jmath}\right\}+K$. But multiplication by integers $n_{1}$ on $B_{1}$ and $n_{2} \neq n_{1}$ on $B_{2}$ is an $E$-map of $G / K$ to $G / K$ which is not induced by a map in $Z E$. By Lemma 1.2, this is a contradiction.

Now suppose $t_{t_{1}} \leqq t_{t^{2}} \lesseqgtr \cdots$ is a countable descending chain of types in $T$ which is not bounded below. Let $p$ be a prime not dividing $A_{t 1}$ and define $K$ to be sum of $\left\{A_{l} \mid t_{l} \not t_{t l}\right\}$ and $\left\{p^{k} A_{l} \mid t_{l} \leqq t_{t k}, t_{l} \equiv t_{l k+1}, \quad k=\right.$ $1,2, \cdots\}$. Then $K$ is fully invariant and $G / K$ is an unbounded torsion group. Since $G$ is $E$-indecomposable, by Lemmas 2.2 and 1.3 , this is a contradiction.

Finally, assume $t_{0} \in T$ is finite at an infinite set of positions $\left\{p_{\jmath}\right\}$, and suppose no $t_{t} \leqq t_{0}$ is zero on an infinite subset of $\left\{p_{j}\right\}$. Then for each $t_{t} \leqq t_{1}$, choose $x_{t} \in A_{t}$ such that $p_{\jmath}$-height $x_{t} \geqq 1$ for all $p_{l}$. Now let $H$ be the minimal fully invariant subgroup containing the $x_{1}$. Since homomorphisms do not decrease height, $\left(1 / p_{l}\right) x_{0} \notin H$ for any $p_{r}$. Thus $A_{0} / A_{0} \cap H$ contians a copy of $Z\left(p_{l}\right)$ for each $p_{\jmath}$. By Lemma 1.3 this is a contradiction.

(2) $\Rightarrow(3)$. Let $H$ be fully invariant in $G$ such that $G / H$ is torsion. Suppose first that for some $A_{l}, A_{i} / A_{i} \cap H$ is unbounded, with nonzero $p_{k}$-component for an infinite set $P=\left\{p_{k}\right\}$ of primes. Note that $A_{\imath} / A_{\imath} \cap H$ contains no $Z\left(p^{\infty}\right)$ since $\operatorname{rank} A_{\imath}=1$ and $A_{\imath} \cap H$ is fully invariant in $A_{l}$. We may, therefore, assume that $t_{t}$ is finite and positive at all $p_{k} \in P$. By condition 2(c), there exists $t_{l}<t_{t}$ such that $t_{l}$ is zero at an infinite subset of $P$. Since $H$ is fully invariant and $t_{j}<t_{t}, A_{i} \cap H \subseteq$ $p_{k} A_{\imath}$ implies $A, \cap H \subseteq p_{k} A_{\text {, for all }} p_{k} \in P$. This is impossible since $t_{j}$ is zero at infinitely many $p_{k}$.

Now if $G / H$ is unbounded, choose a countable sequence $A_{t_{1}}, A_{t_{2}}, \cdots$ such that $\oplus \sum_{r=1}^{\infty} A_{l_{r}} / A_{t_{r}} \cap H$ is unbounded. By conditions 2(a) and 2(b) there exists a fixed $A_{t}$ with $t_{t} \leqq t_{t r}$, for all $r \geqq 1$. It follows that $A_{t} / A_{i} \cap H$ must be unbounded. This is impossible, as above.

(3) $\Rightarrow(1)$. It is easy to show that if $G / K$ is bounded for all fully invariant $K$ with $G / K$ torsion, then $\operatorname{Hom}_{E}(G, G / K)=\{n \Pi \mid n \in Z\}$ where $\Pi: G \rightarrow G / K$ is the natural factor map. It follows that $\operatorname{Hom}_{g}(G, G / K)=\{n \Pi \mid n \in Z\}$, for any fully invariant $K$.

The above theorem characterizes the completely decomposable Eqp groups since any completely decomposable group $G$ may be expressed as a direct sum $\oplus \Sigma G_{1}$ of $E$-indecomposable subgroups which are completely decomposable, and in this decomposition End $\left(G_{i}\right)=\left.E\right|_{G_{i}}$.

COROllary 2.1. Let $G$ be completely decomposable of finite rank with type set $T$. Then $G$ is Eqp iff $T$ satisfies $2(a)$ and minimal types in $T$ are idempotent. 
Proof. $T$ is finite so that minimal types are idempotent iff 2(c) holds. Since 2(b) holds vacuously, the result follows.

Corollary 2.2. Let $G=\bigoplus_{i \in I} \Sigma A_{i}$ with $\left\{A_{i} \mid i \in I\right\}$ rigid [4]. Then $G$ is Eqp iff $t\left(A_{i}\right)$ is idempotent for all $i \in I$.

Proof. If $\left\{A_{l}\right\}$ is rigid, (a) and (b) hold vacuously and (c) holds iff each $t\left(A_{1}\right)$ is idempotent.

Remark. Since $E$ is commutative if $\left\{A_{1}\right\}$ is rigid, Corollary 2.2 can also be derived from a trivial modification of a result of Arnold ([1], Theorem 1.1).

EXAMPLE. The following is a nontrivial (uncountable $E$ indecomposable) example of a completely decomposable group satisfying 2(a), 2(b) and 2(c) of 2 in Theorem 2.1.

Define a relation on the set $I$ of all infinite subsets of the natural numbers by $S \leqq T$ iff $S \backslash T$ is finite. Let $\left\{S_{\alpha}\right\}$ be a maximal chain in I. It is easy to see that $\left\{S_{\alpha}\right\}$ is uncountable. For each $\alpha$, define a type $t_{\alpha}$ by $t_{\alpha}=\left[\left\langle x_{\imath}^{\alpha}\right\rangle\right] ; x_{1}^{\alpha}=1, i \in S_{\alpha} ; x_{1}^{\alpha}=0, i \notin S_{\alpha}$. It is easy to see that $\left\{t_{\alpha}\right\}$ satisfies 2(a) and (b) of Theorem 2.1. By the maximality of the chain $\left\{S_{\alpha}\right\},\left\{t_{\alpha}\right\}$ also satisfies 2(c). Let $A=\bigoplus \Sigma_{\alpha} A_{\alpha}$, where $A_{\alpha}$ is of rank one and type $t_{\alpha}$. Then $A$ is Eqp by Theorem 2.1.

We next characterize homogeneous separable Eqp groups ([4], §87).

LEMMA 2.3. Let $G$ be homogeneous and separable. Then $Z E \subseteq Q$.

Proof. This is an easy exercise. (See [4], Problem 12, page 235.)

Lemma 2.4. Let $G$ be homogeneous and separable. Then, for all nonzero fully invariant $K \subseteq G$, we have $G / K$ is a torsion group.

Proof. Let $0 \neq K$ be fully invariant in $G$. Choose $0 \neq x \in$ $K$. Since $G$ is homogeneous separable we can write $G=\langle x\rangle_{*} \oplus G^{\prime}$, where $\langle x\rangle_{*}$ denotes the pure subgroup generated by $x$. If $G^{\prime} \subseteq K$, then $G / K=\langle x\rangle_{*} /\langle x\rangle_{*} \cap K$ and $G / K$ is torsion. Otherwise, choose $y \in$ $G^{\prime} \backslash G^{\prime} \cap K$. Since $G^{\prime}$ is also homogeneous separable, write $G=$ $\langle x\rangle_{*} \oplus\langle y\rangle_{*} \oplus G^{\prime \prime}$. Since $G$ is homogeneous, there exists $\alpha \in E, n \in Z^{+}$ with $\alpha(x)=n y$. Thus, $n y \in K$. Since $y$ was an arbitrary element of $G^{\prime} \backslash G^{\prime} \cap K$, we have $G / K=\langle x\rangle_{*} /\langle x\rangle_{*} \cap K \oplus G^{\prime} / G^{\prime} \cap K$ is torsion.

Remark. The claim made in Problem 13, page 235 of [4] is incorrect. Any rank one group of nil type will ser e as a counterexample. 
A group $G$ is called strongly irreducible iff for all nonzero fully invariant $K \subseteq G, G / K$ is bounded. (See [7].)

THEOREM 2.2. Let $G$ be a homogeneous separable group. Then $G$ is Eqp iff $G$ is strongly irreducible.

Proof. If $G$ is homogeneous, separable and Eqp, Lemmas 1.3, 2.3 and 2.4 show that $G$ is strongly irreducible.

Conversely, let $G$ be strongly irreducible, homogeneous and separable. Let $K \neq(0)$ be fully invariant in $G$ and $\theta \in$ $\operatorname{Hom}(G, G / K)$. Write $G / K$ in its primary decomposition, $G / K=$ $\bigoplus \sum_{i=1}^{N}(G / K)_{p_{i}}$. Say, for some fixed $p_{j} \in\left\{p_{i} \mid i=1 \cdots N\right\}$, we have $(G / K)_{p_{1}}=\bigoplus \Sigma_{\alpha \in A_{1}}\left\langle\bar{a}_{\alpha}\right\rangle$ with order $\left(\bar{a}_{\alpha}\right)=p_{l}^{s_{\alpha}}, \quad s_{\alpha} \leqq s_{\text {J }} \quad$ (Here $\bar{a}=$ $a+K)$. Since $\theta$ is an $E$ map and $G$ is homogeneous separable, it is easy to show that, for some fixed $m_{\jmath} \in Z^{+}$, we must have $\theta\left(a_{\alpha}\right)=m_{\jmath} \bar{a}_{\alpha}$ for all $\alpha \in A_{j}$. Choose $m \in Z^{+}$with $m \equiv m_{j}\left(p_{j}^{s_{j}}\right), j=1 \cdots N$. Then $\Pi m=$ $\theta$.

The final results of this section deal with groups $G$ which can be written as a sum of two groups related in a special way. We will need the notions of outer type (OT) and inner type (IT) of a group as defined in Warfield [8].

THEOREM 2.3. Let $G=A \oplus B$ where $\operatorname{IT}(A)>O T(B)$ and let $\bar{E}=$ $\operatorname{End}(B)$. Then $G$ is Eqp iff $B$ is $\bar{E} q p$ and $\operatorname{rank} Z \bar{E}=1$.

Proof. $\quad(\Rightarrow)$ Let $K$ be an $\bar{E}$-submodule of $B$. Then $A \oplus K$ is an $E$-submodule of $G$ since $\operatorname{Hom}(A, B)=0$. Therefore, any $\bar{E}$-map $\theta: B \rightarrow B / K$, induces an $E$-map $0 \oplus \theta: A \oplus B \rightarrow A \oplus B / A \oplus K$ which must lift to a map in $Z E$ of the form $\alpha \oplus \beta$, where $\alpha: A \rightarrow A$, $\beta: B \rightarrow B$. It follows that $\beta$ is an $\bar{E}$-map which lifts $\theta$.

Now suppose rank $Z \bar{E}>1$. Choose $\gamma \in Z \bar{E}$ and $b \in B$, such that $b, \gamma(b)$ are independent. Then $0 \oplus \gamma: A \oplus B \rightarrow(A \oplus B) / A$ is an $E$ map and lifts as above to a map of the form $\alpha \oplus \beta$ in ZE. Since $I T(A)>O T(B)$, there exists $\delta \in \operatorname{Hom}(B, A)$ such that $\delta(b)=0$ and $\delta(\gamma(b)) \neq 0$. But then $0=\alpha \delta(b)=\delta \beta(b)=\delta \gamma(b) \neq 0$, a contradiction.

$(\Leftarrow)$ Let $K$ be a fully invariant subgroup of $G$, and $\theta: G \rightarrow G / K$ an $E$-map. Then $K=K \cap A \oplus K \cap B$ and $\theta(B) \subseteq B / B \cap K$ so that $\theta$ restricted to $B$ may be lifted to a map $\alpha \in Z \bar{E} \subseteq Q$. Since $I T(A)>$ $O T(B), A=\bigcup_{f . B \rightarrow A}$ Image $f . \quad$ It follows that $\alpha: A \rightarrow A$ must be a lifting of $\left.\theta\right|_{A}$.

REMARK. This theorem may be generalized slightly to the case $I T(A) \geqq O T(B)$. 
Corollary. If $G=D \oplus R$ where $D$ is divisible and $R$ is reduced, then $G$ is Eqp iff $R$ is $E(R) q p$ and rank $Z E(R)=1$.

3. Strongly indecomposable groups. In this section we characterize the strongly indecomposable Eqp groups of finite rank. We start by characterizing the strongly indecomposable, strongly irreducible ones. Recall that a group $G$ is called strongly indecomposable if it admits no nontrivial quasi-decompositions ([4], §92).

THEOREM 3.1. Let $G$ be strongly indecomposable, strongly irreducible of finite rank. Then $G$ is Eqp iff $G / P^{k} G$ is a cyclic $E$ module for all nonzero prime ideals $P \subseteq E$.

Proof. Suppose $G$ is Eqp. Since $G$ is strongly indecomposable and strongly irreducible, we can conclude that $E$ is a subring of an algebraic number field $F$ with $Q E=F$. (See [7].) Note that $E$ is Noetherian and $P \neq(0)$ prime in $E$ implies $P$ is maximal. (Since $Q E=F$, every nonzero ideal $I \subseteq E$ contains a nonzero rational integer. Thus, $E / I$ is finite.)

We show $G / P^{k} G$ is a cyclic $E$ module for all nonzero prime ideals $P \subseteq E$. If not, let $X=\left\{\bar{x}_{1} \cdots \bar{x}_{n}\right\}$ be a minimal set of $E$ generators for $G / P^{k} G$, where $\bar{x}_{i}=x_{1}+P^{k} G$. Let $H$ be given by $E \bar{x}_{1} \cap \sum_{i=2}^{n} E \bar{x}_{i}=$ $H / P^{k} G$. Then $H$ is fully invariant and $G / H=A \oplus B$ with $A=E \overline{\bar{x}}_{1}$, $B=\sum_{i=2}^{n} E \overline{\bar{x}}_{i}$, where $\overline{\bar{x}}_{i}=x_{i}+H$. This is a nontrivial direct sum decomposition because of the minimality of $X$.

Let $f, g$ be the projections from $G / H$ onto $A, B$ and $\Pi: G \rightarrow G / H$ the natural map. Let $\bar{f}, \bar{g} \in E$ be such that $\Pi \bar{f}=f \Pi, \quad \Pi \bar{g}=$ gП. Finally, let $I=\{\alpha \in E \mid \alpha(G) \subseteq H\}$. Then $P^{k} \subseteq I$, so $I \subseteq p$ (primes in $E$ are maximal). Clearly $\overline{\bar{f}} \in I \subseteq P$, so $\bar{f} \in P$ or $\bar{g} \in P$. If $f \in P, P A=A$. Thus, $P^{k} A=A$, so $I A=A$. But $I A=(0)$ and $A \neq(0)$ - a contradiction. A similar contradiction arises from the assumption $\bar{g} \in P$. Thus $G / P^{k} G$ is cyclic.

Conversely, let $G$ be strongly indecomposable strongly irreducible of finite rank with $G / P^{k} G$ cyclic for all nonzero primes $P \subseteq E$. We show, for all positive rational integers $n, G / n G$ is $E$ cyclic. Let $n \in Z^{+}$. Since $(0) \neq(n) \subseteq E$ and $E$ is Noetherian we have $(n) \supseteq$ $P_{1}^{k_{1}} \cdots P_{s}^{k_{s}}$ with the $P_{i}$ 's nonzero prime ideals in $E$ ([10], page 200). Now the ideals $P_{i}^{k_{i}}, i=1 \cdots s$, are co-maximal in $E$ ([9], page 176) and, by assumption, $G / P_{l}^{k_{l}} G$ is $E$-cyclic. It is easy to show (using the Chinese Remainder Theorem in $E$ ) that $G /\left(\Pi P_{1}^{k_{i}}\right) G$ is $E$-cyclic. Thus, $G / n G$ is E-cyclic.

Now let $\theta: G \rightarrow G / K$ be an $E$ map, $(0) \neq K$ a fully invariant subgroup of $G$. Since $G$ is strongly irreducible, $n G \subseteq K$ for some positive integer $n$. Thus $G / K$ is $E$-cyclic, say $G / K=$ 
$E(g+K)$. Choose $\alpha \in E$ with $\theta(g)=\alpha(g+K)$. We claim that $\alpha$ is a lifting of $\theta$. To show this, it only remains to show that $\theta(K)=(0)$. Let $G / n G=E(h+n G)$. Then for any $k \in K, k=\beta h+n x$ for some $\beta \in E, x \in G$. Now $\beta h \in K$, so, since $E$ is commutative, $\beta(G) \subseteq$ $K$. Thus, $\theta \beta(G)=\beta \theta(G)=(0)$ in $G / K$. Finally, $\theta(k)=$ $\theta \beta(h)+n \theta(x)=0+K$. This shows that $G$ is Eqp and completes the proof.

We now consider the general case, and begin with a more general definition of quasi-projectivity which is invariant under quasiisomorphism.

Definition. If $R$ is a ring, an $R$-module $M$ is almost quasiprojective, if there exists a positive integer $t$ such that the image of $\operatorname{Hom}_{R}(M, M)$ in $\operatorname{Hom}_{R}(M, M / N)$ is bounded by $t$ for every submodule $N$ of $M$.

LemMA 3.1. If $M \sim N$ are (quasi-isomorphic) $R$ modules, and $M$ is almost quasi-projective, then $N$ is almost quasi-projective.

Proof. Without loss of generality, assume $n M \subseteq N \subseteq M$ for some positive integer $n$. Let $K$ be a submodule of $N$ and $f: N \rightarrow N / K$. Then $n f: M \rightarrow M / K$ lifts to a map $\bar{f} \in \operatorname{Hom}_{R}(M, M)$ such that $\Pi \bar{f}=\operatorname{tnf}$ where $\Pi: M \rightarrow M / K$. Then $n \bar{f} \in \operatorname{Hom}_{R}(N, N)$ and is a lifting of $\operatorname{tn}^{2} f$. Hence $N$ is almost quasi-projective.

LEMMA 3.2. Let $G$ be strongly indecomposable and almost Eqp. Then there is a $g \in G$ such that $G / E g$ is bounded.

Proof. Choose $\left\{g_{1}, \cdots, g_{k}\right\}$ of minimal cardinality with respect to $G / E g_{1}+E g_{2}+\cdots+E g_{k}$ is bounded. This is possible by Lemma 1.3. If $k>1$, let $H=E g_{1} \cap \sum_{i=2}^{k} E g_{1}$. Then $H$ is fully invariant and $\sum_{l=1}^{k} E g_{\imath} / H=E g_{1}+H \oplus \sum_{i=2}^{k} E g_{1}+H$. Furthermore, $E g_{1}+H$ is not torsion since $n g_{1} \in H \Rightarrow n E g_{1} \subseteq H$, contradicting the minimality of $k$. Since $G$ is strongly indecomposable, any $\alpha \in E$ is either monic or nilpotent (see [6]). But if $t$ is a positive integer such that $t G \subseteq \sum_{l=1}^{k} E g_{l}$, then $t$ followed by projection onto $\sum_{\imath=2}^{k} E g_{\imath}+H$ is a map from $G$ to $G / H$ which cannot be lifted, as the lifting could be neither monic nor nilpotent. Thus $k=1$, proving the lemma.

THEOREM 3.2. Let $G$ be strongly indecomposable of finite rank. Then $G$ is Eqp iff $G$ is strongly irreducible and $G / P^{k} G$ is a cyclic $E$ module for all nonzero prime ideals $P \subseteq E$. 
Proof. In view of Theorem 3.1, we only need show that strongly indecomposable Eqp groups of finite rank are strongly irreducible.

By the preceding Lemma $n G \subseteq E g \subseteq G$ for some $n$ and $E g \cong E / L$ (as $E$-modules) for some left ideal $L \subseteq J(E)$, the Jacobson radical of $E$. Therefore by Lemma $3.1, E / L$ is almost Eqp, with associated integer $t$, for some $t>0$.

Now for any $x \in E$ consider

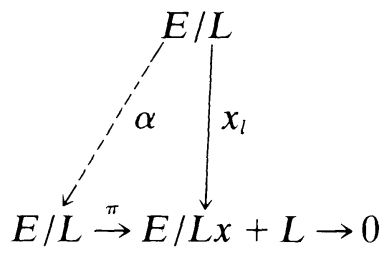

$$
\begin{aligned}
& x_{l}=\text { left multiplication by } x \\
& L x+L \subseteq J(E) \neq E .
\end{aligned}
$$

Then $n \alpha$ is an $E$ endomorphism of $G$, hence in $Z E$. Furthermore $n \alpha-t x_{l}: E \rightarrow L x+L$, so that $n \alpha-t x \in L x+L$. Hence $t x \in Z E+$ $L x+L$. This implies $L t x \subseteq L+L^{2} x$, so that $t^{2} x \in Z(E)+L^{2} x+$ $L$. Continuing inductively $t^{k} x \in Z(E)+L^{k} x+L$. Since $L$ is nilpotent $(L \subseteq J(E))$, for some $m>0, \quad L^{m}=0$ and $t^{m} x \in Z E+L$. Thus $t^{m} E \subset Z(E)+L$, and $G \sim E / L \sim Z(E)+L / L \cong Z E / L \cap Z E$, a commutative ring with identity. By ([2], Th. 1.4, Cor. 3.6, Th. 1.13) $G$ must be strongly irreducible.

COROllary 3.1. Let $G$ be finite rank strongly indecomposable with $\operatorname{rank} E<\operatorname{rank} G$. Then $G$ is not Eqp.

Proof. For any $0 \neq g \in G, E g$ is a fully invariant subgroup of $G$ with $\operatorname{rank} E g \leqq \operatorname{rank} E<\operatorname{rank} G$. Thus, $G$ is not strongly irreducible, so $G$ cannot be Eqp.

4. Groups of rank two. In this section we use the results of $\S \S 1-3$ to survey the Eqp property for groups of rank two. This is most conveniently done by considering the six possibilities for the quasiendomorphism algebra, $Q E(G)=Q \otimes_{z} E(G)$. (See [3].) If $Q E(G) \cong$ $[Q]_{2 \times 2}$ or $Q E(G) \cong\left\{\left(\begin{array}{ll}x & 0 \\ y & z\end{array}\right) \mid x, y, z \in Q\right\}$ then $G$ is completely decomposable. In the first case we have $G=A \oplus A$, and in the second case $G=A \oplus B$ with $A, B$ of rank one, $t(A)<t(B)$. In either case Corollary 2.1 applies; $G$ is Eqp iff $t(A)$ is idempotent. If $Q E(G) \cong$ $Q \oplus Q$, then $G$ is quasi-decomposable $G \sim A \oplus B$ with $t(A), t(B)$ incomparable. A slight modification of the arguments of Theorem 2.1 prove that $G$ is Eqp iff $t(A)$ and $t(B)$ are idempotent.

We next consider the strongly indecomposable cases. If $Q E(G) \cong$ 
$Q$ or $Q E(G) \cong\left\{\left(\begin{array}{ll}x & 0 \\ y & x\end{array}\right) \mid x, y \in Q\right\}$, then $G$ is strongly indecomposable but not strongly irreducible, so $G$ is not Eqp by Theorem 3.2. We settle the final possibility, $Q E(G) \cong Q(\sqrt{N})$, in the following theorem.

THEOREM 4.1. Let $G$ be of rank two with $Q E(G) \cong Q(\sqrt{N})$. Then $g$ is Eqp iff $G$ is strongly irreducible.

Proof. If $G$ is Eqp, $G$ is strongly irreducible by Theorem 3.2. Conversely, let $G$ be strongly irreducible and $K$ any nonzero fully invariant subgroup of $G$. Write the finite group $G / K$ in its primary decomposition: $G / K=\bigoplus_{i=1}^{n}(G / K)_{p_{i}}$. Since rank $G=2, K$ is fully invariant, and $Q E(G)=Q(\sqrt{N})$, it is easy to show, for each $p_{\imath}$, either $\left(G / K_{l}\right)_{p_{t}}=Z\left(p_{i}^{s_{i}}\right)$ for some $s_{l} \geqq 0$ in $Z$, or $\left(G / K_{i}\right)_{p_{1}}=Z\left(p_{i}^{t_{i}}\right) \oplus Z\left(p_{i}^{t_{i}}\right)$ for some $t_{t} \geqq 0$ in $Z$. Moreover, in the latter case we can choose $a \in G$ so that $a+K_{t}$ and $\sqrt{N} a+K_{t}$ are generators of $\left(G / K_{t}\right)_{p_{t}}$. It is now easy to check that $G / K$ is a cyclic $E$ module. Thus, Theorem 3.2 applies and $G$ is Eqp.

\section{REFERENCES}

1. D. M. Arnold and E. L. Lady, Endomorphism Rings and Direct Sums of Torsion Free Abelian Groups, Trans. Amer. Math. Soc., 211 (1975), 225-237.

2. R. A. Beaumont and R. S. Pierce, Torsion free rings, Illinois J. Math., 5 (1961), 61-98.

3. - Torsion Free Groups of Rank Two, Amer. Math. Soc., Mem. 38, A. M. S., Providence, 1961.

4. L. Fuchs, Infinite Abelian Groups, Vol. II, Academic Press, New York, 1973.

5. - On Torsion Abelian Groups Quasi-Projective over Their Endomorphism Rings, Proc. Amer. Math. Soc., 42, No. 1, Jan. (1974), 13-15.

6. J. D. Reid, On the Ring of Quasi-Endomorphisms of a Torsion Free Group, Topics in Abelian Groups, Chicago, 1963, 51-58.

7. - Remarks on irreducible groups, preprint.

8. R. B. Warfield, Jr., Homomorphisms and duality for torsion-free Abelian groups, Math. Z., 107 (1968), 189-200.

9. L. E. T. Wu and J. P. Jans, On Quası Projectives, Illinois J. Math., 11 (1967), 439-448.

10. O. Zariski and P. Samuel, Commutative Algebra, Vol. I, Van Nostrand, Princeton, 1958.

Received May 3, 1976.

UNIVERSITY OF CONNECTICUT

STORRS, CT 06268 



\section{PACIFIC JOURNAL OF MATHEMATICS}

\section{EDITORS}

RichaRd ARENS (Managing Editor)

University of California

Los Angeles, CA 90024

\section{R. A. Beaumont}

University of Washington

Seattle, WA 98105

C. C. Moore

University of California

Berkeley, CA 94720
J. DugunduI

Department of Mathematics

University of Southern California

Los Angeles, CA 90007

R. Finn AND J. Milgram

Stanford University

Stanford, CA 94305

\section{ASSOCIATE EDITORS}
E. F. BECKENBACH
B. H. NeumanN
F. WOLF
K. YoshidA

\section{SUPPORTING INSTITUTIONS}

UNIVERSITY OF BRITISH COLUMBIA

CALIFORNIA INSTITUTE OF TECHNOLOGY

UNIVERSITY OF CALIFORNIA

MONTANA STATE UNIVERSITY

UNIVERSITY OF NEVADA

NEW MEXICO STATE UNIVERSITY

OREGON STATE UNIVERSITY

UNIVERSITY OF OREGON

OSAKA UNIVERSITY

\author{
UNIVERSITY OF SOUTHERN CALIFORNIA \\ STANFORD UNIVERSITY \\ UNIVERSITY OF HAWAII \\ UNIVERSITY OF TOKYO \\ UNIVERSITY OF UTAH \\ WASHINGTON STATE UNIVERSITY \\ UNIVERSITY OF WASHINGTON \\ AMERICAN MATHEMATICAL SOCIETY
}

The Supporting Institutions listed above contribute to the cost of publication of this Journal, but they are not owners or publishers and have no responsibility for its contents or policies.

Mathematical papers intended for publication in the Pacific Journal of Mathematics should be in typed form or offset-reproduced (not dittoed), double spaced with large margins. Underline Greek letters in red, German in green, and script in blue. The first paragraph or two must be capable of being used separately as a synopsis of the entire paper. Items of the bibliography should not be cited there unless absolutely necessary, in which case they must be identified by author and Journal, rather than by item number. Manuscripts, in duplicate, may be sent to any one of the four editors. Please classify according to the scheme of Math. Reviews, Index to Vol. 39. All other communications should be addressed to the managing editor, or Elaine Barth, University of California, Los Angeles, California, 90024.

100 reprints are provided free for each article, only if page charges have been substantially paid. Additional copies may be obtained at cost in multiples of 50 .

The Pacific Journal of Mathematics is issued monthly as of January 1966. Regular subscription rate: $\$ 72.00$ a year (6 Vols., 12 issues). Special rate: $\$ 36.00$ a year to individual members of supporting institutions.

Subscriptions, orders for back numbers, and changes of address should be sent to Pacific Journal of Mathematics, 103 Highland Boulevard, Berkeley, California, 94708.

PUBLISHED BY PACIFIC JOURNAL OF MATHEMATICS, A NON-PROFIT CORPORATION

Printed at Jerusalem Academic Press, POB 2390, Jerusalem, Israel. 


\section{Pacific Journal of Mathematics}

Vol. 68, No. 2

April, 1977

William Allen Adkins, Aldo Andreotti and John Vincent Leahy, An analogue of Oka's theorem for weakly normal complex spaces ........

Ann K. Boyle, M. G. Deshpande and Edmund H. Feller, On nonsingularly

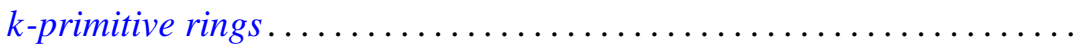

Rolando Basim Chuaqui, Measures invariant under a group of

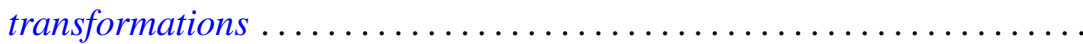

Wendell Dan Curtis and Forrest Miller, Gauge groups and classification of bundles with simple structural group .......................

Garret J. Etgen and Willie Taylor, The essential uniqueness of bounded nonoscillatory solutions of certain even order differential equations

Paul Ezust, On a representation theory for ideal systems

Richard Carl Gilbert, The deficiency index of a third order operator ........

John Norman Ginsburg, $S$-spaces in countably compact spaces using Ostaszewski's method.

Basil Gordon and S. P. Mohanty, On a theorem of Delaunay and some

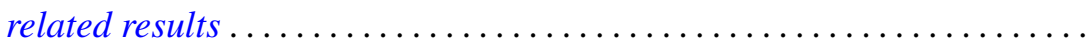

Douglas Lloyd Grant, Topological groups which satisfy an open mapping

theorem.

Charles Lemuel Hagopian, A characterization of solenoids

Kyong Taik Hahn, On completeness of the Bergman metric and its

subordinate metrics. II .

G. Hochschild and David Wheeler Wigner, Abstractly split group extensions.

Gary S. Itzkowitz, Inner invariant subspaces ...............

Jiang Luh and Mohan S. Putcha, A commutativity theorem for

non-associative algebras over a principal ideal domain.

Donald J. Newman and A. R. Reddy, Addendum to: "Rational approximation of $e^{-x}$ on the positive real axis".....

Akio Osada, On the distribution of a-points of a strongly annular function ....

Jeffrey Lynn Spielman, A characterization of the Gaussian distribution in a Hilbert space. .

Robert Moffatt Stephenson Jr., Symmetrizable-closed spaces ...

Peter George Trotter and Takayuki Tamura, Completely semisimple inverse $\Delta$-semigroups admitting principal series . . . . . . . .

Charles Irvin Vinsonhaler and William Jennings Wickless, Torsion free abelian groups quasi-projective over their endomorphism rings...

Frank Arvey Wattenberg, Topologies on the set of closed subsets ... 